\title{
Regional influences on the dispensing of glucose test strips in Dutch community pharmacies
}

\author{
Michiel J. Storimans • Olaf H. Klungel • \\ Herre Talsma $\cdot$ Cornelis J. de Blaey
}

Received: 10 December 2004 / Accepted: 28 August 2005 / Published online: 15 May 2006

(C) Springer Science + Business Media B.V. 2006

\begin{abstract}
Background: Pharmacy practice guidelines promote the role of community pharmacies in selfmonitoring of blood glucose. However, variations between Dutch pharmacies exist in the proportion of patients to whom test strips are dispensed. Objective: To assess whether variations between community pharmacies in dispensing of blood glucose test strips can be explained by differences in patient characteristics and the region in which the pharmacy is located. Setting: PHARMO-Record Linkage System containing drug dispensing histories from 40 community pharmacies of about 450,000 patients in the Netherlands. Method: We performed a retrospective cohort study. Data on prescription of all drugs and medical aids between 1991 and 2001 were extracted for all new users of antidiabetic drugs. Patients were excluded if the dispensing history did not allow classification of the type of diabetes or if the dispensing pharmacy could not be determined. The data were analysed using a Cox proportional hazard model. Main outcome measure: Time to first test strips dispensed. Results: We identified 8,233 starters of antidiabetic drugs. During a median follow-up of 2.1 years, $20 \%$ of the patients were dispensed test strips at least once. Community pharmacy was significantly associated with the
\end{abstract}

Parts of this study have been published in Ann Pharmacother 2004: 38: 1751-1752.

M. J. Storimans $\cdot$ O. H. Klungel $(\bowtie) \cdot H$. Talsma •

C. J. de Blaey

Department of Pharmacoepidemiology and pharmacotherapy, Utrecht Institute for Pharmaceutical Sciences, Utrecht University, P.O. Box 80082, 3508 TB, Utrecht,

The Netherlands

E-mail: o.h.klungel@pharm.uu.nl

Tel.: + 31-(0)30-253-6987

Fax: + 31-(0)30-2539166

M. J. Storimans · C. J. de Blaey

Scientific Institute Dutch Pharmacists, The Hague, The Netherlands dispensing of test strips after adjustment for patient characteristics. This association was less apparent when stratified for the geographical location of the pharmacy. Conclusion: Community pharmacy is an independent determinant of the start of use of test strips. Differences in dispensing of test strips between pharmacies are dependent on geographical region. This suggests that implementing practice guidelines for diabetes care in community pharmacy requires different approaches in different regions.

Key words Cohort analysis - Diabetes mellitus · Blood glucose monitoring - Pharmaceutical care · Netherlands · Glucose test strips

Diabetes is a chronic illness that requires continuing medical care and patient self-management to prevent acute complications and to decrease the risk of longterm complications. Reducing hyperglycemia has been shown to be an important factor in the prevention of diabetic complications $[1,2]$. Besides pharmacological treatment, education on self-management of diabetes is considered an integral component of all diabetes care plans $[3,4]$.

An important aspect of self-management is the selfmonitoring of blood glucose (SMBG). SMBG enables both type 1 and type 2 diabetic patients to achieve and maintain specific individual glycemic goals [5]. National and international pharmacy practice guidelines recognise this and promote the role of community pharmacy in supporting patients performing SMBG [6-9].

In a previous study, we found large differences in the rate of dispensing of test strips among pharmacies in the Netherlands [10]. This finding may indicate that not all pharmacies are able to implement the guidelines to the same extent. However, community pharmacies may differ substantially in their diabetic patient population (i.e., age, proportion of type 1 versus type 2 patients). It has been shown that patient characteristics 
are determinants of test strip use [11, 12]. Furthermore, regional differences may also be important to consider when comparing the diabetes care process. This is underscored by the results of the studies by Arday et al., who examined variation in diabetes care among states in the US. Variations in, for example, the rate of annual HbAlc-testing, were significantly reduced when adjusted for differences in patient characteristics. Still, much variability remained unexplained, which might be due to differences in state characteristics [13].

In this study, we assess whether variations between community pharmacies in dispensing of blood glucose test strips can be explained by patient characteristics and regional effects. Differences between pharmacies are important to study in order to identify barriers in dispensing of test strips which in turn may help to design and guide interventions to improve quality of diabetes care.

\section{Methods}

\section{Setting}

We used data from the PHARMO-Record Linkage System (RLS) covering the period 1991-2001. The PHARMO-RLS has been described in detail elsewhere $[14,15]$. In brief, the system includes pharmacy dispensing records from community pharmacies linked to hospital discharge records of all 450,000 communitydwelling residents of nine population-defined areas in the Netherlands from 1985 onwards. Since most patients in the Netherlands are registered with a single community pharmacy, independent of prescriber, pharmacy records are virtually complete with regard to prescription drugs. Medical aid dispensing records may be less complete, because SMBG-equipment can be dispensed without a prescription. Still, a prescription is necessary for remuneration of test materials. Hence, in most cases dispensing of test strips is recorded in the patient's medication history.

Drug use was coded according to the Anatomical Therapeutic Chemical (ATC) classification index of the World Health Organization. Since not all pharmacies use the standard index for SMBG-equipment, a patient's complete dispensing history was reviewed. Dispensing records of test strips were selected based on the text description in the label-field together with the number of units dispensed.

\section{Study design and population}

We performed a retrospective cohort study among new users of antidiabetic drugs. All diabetes mellitus patients who filled at least two prescriptions of an antidiabetic drug (ATC-codes A10A or A10B) in the period of 1991-2001 were included in this study. Patients with less than 365 days of medication history before the first prescription of an antidiabetic drug were excluded (non-incident patients). Patients with gestational diabetes, defined as women younger than 50 years using insulin for less than 210 days with no prescription of an antidiabetic drug for at least 1 year after discontinuing insulin treatment, were also excluded. Type 1 diabetes mellitus was defined as at least two prescriptions of insulin, no more than one prescription of an OHA and not older than 50 years at the start of insulin therapy. Type 2 diabetes mellitus was defined as at least two prescriptions of an OHA. A similar definition was used in previous studies with data from the PHARMO-RLS [16]. Patients of whom the type of diabetes could not be classified were excluded.

\section{Data collection}

Of all patients in the study population $(n=8,233)$, the dispensing histories of drugs and diabetes test materials were extracted from the database from the period of January 1991 until December 2001. Retrievable information per prescribed drug and medical aid included date of dispensing, drug name, dosage, number of prescribed dosage units and dispensing pharmacy. Patient information included gender and year of birth.

For all patients, the number of prescriptions of antidiabetic drugs per pharmacy was determined. Patients who collected antidiabetic drugs in two or more pharmacies were only included if they visited one of those pharmacies on at least three occasions to collect an antidiabetic drug. Furthermore, the patient had to fill at least $85 \%$ of all antidiabetic drug prescriptions in that pharmacy. The dispensing pharmacy was defined as the pharmacy in which the patient collected the most prescriptions of antidiabetic drugs.

From the dispensing records, the type of antidiabetic drug treatment (oral hypoglycemic agents, insulin or both) was recorded as a time-dependent variable.

Furthermore, data on use of chronic co-medication in the year prior to the first prescription of antidiabetic drug was collected. Use of co-medication was defined as at least one prescription of a drug in the year before the index date.

\section{Analysis}

Follow-up started at the first prescription of an antidiabetic drug and lasted until the first test strip was dispensed or at the end of follow-up, whichever came first. The Cox proportional hazard model was used to determine the association between the determinants and the time to the first test strip dispensed. The effect 
of dispensing pharmacy was analysed separately by introducing it to a hazard model containing all patient characteristics. Finally, we assessed whether the effect of dispensing pharmacy was modified by the geographical region in which the pharmacy was located.

The hazard ratio of dispensing pharmacy was determined using one pharmacy as reference. This pharmacy's patient population showed the least deviation from the mean age, the total number of patients and the mean proportion of men. In the stratified analysis for geographical region, the reference pharmacy was the pharmacy with the most incident users of antidiabetic drugs in that region.

Analyses were performed using S-PLUS 6 Professional Edition for windows, release 2.

\section{Results}

A total of 18,128 patients in the study population filled at least two prescriptions of antidiabetic drugs between January 1991 and December 2001. After exclusion of non-incident users and patients with gestational diabetes, the number of incident users of antidiabetic drugs was 8,878 . Ninety-four patients were excluded, because data did not allow classification of the dispensing pharmacy. Furthermore, another 551 patients were excluded because the type of antidiabetic drug treatment could not be determined (224 patients with a total of less than three prescriptions of antidiabetic drugs, all dispensed on the same day; 227 patients who filled at least two prescriptions of insulin, did not receive OHAs and were 50 years or older at start of follow-up).

Table 1 presents the patient characteristics of the final study population. A total of 8,233 patients were included (230 type 1 and 8,003 type 2). The median age of the type 1 and type 2 patients was 33 years and 65 years, respectively. The proportion of men in type 1 patients was $59.1 \%$, for type 2 this was $47.5 \%$. The number of incident users increased in the period 1992-2001, especially among type 2 patients. Use of co-medication in the year prior to their start of antidiabetic drug treatment was much higher in type 2 patients. The most commonly used drugs were benzodiazepines and cardiovascular medication (antihypertensives and cholesterol lowering drugs).

\section{Use of test strips}

During follow-up, patients received an average of 39.5 SMBG test strips per year (0.57 occasions of dispensing of test strips per patient per year). Of all 8,233 patients, 1,599 were dispensed test strips at least once $(19.4 \%)$.

\section{Patient characteristics by dispensing pharmacy}

Data were collected in 40 community pharmacies in 17 geographical regions. The median registration period

Table 1 Patient characteristics of incident users $(\%$ of total $)$ of antidiabetic drugs $(n=8233)$, stratified for type of diabetes

\begin{tabular}{lll}
\hline & type 1 patients $(n=230)$ & type 2 patients $(n=8003)$ \\
\hline Age * (median; (IQR)) in years & $33(27)$ & $65(20)$ \\
Proportion men & $136(59.1)$ & $3,802(47.5)$ \\
Year of start of antidiabetic drug use & & $393(4.9)$ \\
1992 & $8(3.5)$ & $570(7.1)$ \\
1993 & $11(4.8)$ & $642(8.0)$ \\
1994 & $17(7.4)$ & $697(8.7)$ \\
1995 & $26(11.3)$ & $708(8.8)$ \\
1996 & $39(17.0)$ & $802(10.0)$ \\
1997 & $27(11.7)$ & $957(12.0)$ \\
1998 & $39(17.0)$ & $1062(13.3)$ \\
1999 & $25(10.9)$ & $1142(14.3)$ \\
2000 & $27(11.8)$ & $1030(12.9)$ \\
2001 & $11(4.8)$ & \\
Use of co-medication (ATC-code) & & $2538(31.7)$ \\
Diuretics (ATC-code: C03, C07B or C07C) & $16(7.0)$ & $2110(26.4)$ \\
Beta blocking agents (C07 except sotalol) & $16(7.0)$ & $1633(20.4)$ \\
ACE-inhibitors and AII antagonists (C09) & $13(5.6)$ & $1265(15.8)$ \\
Calcium channel blockers (C08) & $6(2.6)$ & $1054(13.2)$ \\
Cholesterol lowering drugs (C10A or B04A) & $6(2.6)$ & $869(10.9)$ \\
Systemic corticosteroids (H02) & $9(3.9)$ & $2156(26.9)$ \\
Benzodiazepines (N05BA or N05CD) & $35(15.2)$ & $595(7.4)$ \\
Antidepressants (N06A) & $4(1.7)$ & $263(3.3)$ \\
Antipsychotics (N05A, except N05AN) & $8(3.5)$ & \\
\hline
\end{tabular}

*Age at first prescription of an antidiabetic drug in the PHARMO-RLS database 
of a pharmacy in the database was 11 years (range 3.211 years). Table 2 shows the characteristics of the diabetic population per geographical region and per pharmacy. Although only mean age differed statistically significant between dispensing pharmacy $(P<0.001)$, age, sex and type of diabetes varied between pharmacies and, to a lesser extent, between regions. This variation reflects differences in the setting of the pharmacy, for example neighbourhood and regional demographics.

Determinants for dispensing of test strips in community pharmacy

Age and the year of start of antidiabetic drug use, as well as type of antidiabetic treatment were significantly associated with the time to the first test strips dispensed (Table 3). The hazard ratio (HR) showed that older patients were less likely to receive test strips, also when adjusted for all other characteristics. There were no differences between male and female patients. There was a trend that patients who started antidiabetic drugs after 1997 had a higher chance of receiving test strips in the dispensing pharmacy than before that time. Compared to type 1 patients, patients who used oral hypoglycemic agents (OHAs) were dispensed test strips almost five times less. In contrast, type 2 patients using insulin or both OHAs and insulin collected test strips more often (HR 3.75 [95\% CI: 2.72-5.16] and 1.83 [95\% CI: 1.34-2.50], respectively). Use of specific comedication in the year before the start of antidiabetic drug use was not associated with the outcome.

The variable dispensing pharmacy improved the model $(P<<0.001)$, and was independently associated with starting the use of test strip. Compared to patients in the reference pharmacy, the rate of dispensing of test strips to diabetic patients was significantly different in 14 pharmacies.
Regional effects on dispensing of test strips in community pharmacy

Variation in time to the first test strip dispensed between pharmacies in one region, was considerably less than between pharmacies in different regions (Fig. 1). This indicates that the differences between dispensing pharmacies after adjusting for patient characteristics may also be dependent on the region.

When stratified for region, dispensing pharmacy improves the model in only two out of six regions $(P<0.001$ and $P=0.012)$. In these two regions, there were three pharmacies in which the chance of receiving test strips compared to the reference pharmacy, was statistically significantly different (data not shown).

\section{Discussion}

We found that $20 \%$ of all incident users of antidiabetic drugs ever received test strips through community pharmacies during a median follow-up of 2.1 years. After adjusting for variation in diabetic patient population, differences between pharmacies in dispensing test strips remained. These differences became less apparent when we stratified for geographical region, suggesting that the role of the community pharmacy is significantly modified by the regional structure of care for patients performing self-monitoring.

Several patient characteristics were significantly associated with test strip use. As expected from practice guidelines [5], we found that the type of antidiabetic treatment was most strongly associated with collecting test strips. Type 1 patients were dispensed test strips almost five times more often than patients using only OHAs, but less often than type 2 patients using insulin in combination with OHAs. We also observed that insulin using type 2 patients were more likely to start using test strips compared to type 1 . Our

Table 2 Characteristics (mean $\pm \mathrm{sd}$ ) of diabetic population per pharmacy $(\mathrm{n}=40)$ in 17 geographical regions. Data from regions with only one study pharmacy are pooled

\begin{tabular}{|c|c|c|c|c|c|}
\hline & $\begin{array}{l}\text { no. of pharmacies } \\
\text { (no. patients) }\end{array}$ & age & $\begin{array}{l}\text { proportion } \\
\text { men }(\%)\end{array}$ & $\begin{array}{l}\text { incident type } 1 \\
\text { per year follow-up }\end{array}$ & $\begin{array}{l}\text { incident type } 2 \\
\text { per year follow-up }\end{array}$ \\
\hline overall per region & $40(8233)$ & $62.9(14.5) *$ & $47.8(4.4) \dagger$ & $0.53(0.34) \ddagger$ & $19.1(7.7) \S$ \\
\hline region I & $3(555)$ & $62.4(13.6)$ & $48.6(5.3)$ & $0.42(0.05)$ & $16.4(3.7)$ \\
\hline region $\mathrm{G}$ & $7(1022)$ & $62.5(14.5)$ & $48.5(5.7)$ & $0.30(0.25)$ & $17.4(8.7)$ \\
\hline region $\mathrm{F}$ & $5(1144)$ & $59.1(14.3)$ & $48.8(3.3)$ & $0.44(0.14)$ & $20.4(11.6)$ \\
\hline region $\mathrm{D}$ & $5(1190)$ & $62.8(15.2)$ & $47.3(5.2)$ & $0.77(0.49)$ & $21.0(7.9)$ \\
\hline region $\mathrm{C}$ & $4(1088)$ & $62.8(14.3)$ & $47.8(3.9)$ & $0.62(0.51)$ & $24.9(7.9)$ \\
\hline region $\mathrm{H}$ & $5(686)$ & $65.2(14.9)$ & $45.8(6.0)$ & $0.38(0.30)$ & $12.1(5.6)$ \\
\hline other \| & $11(2548)$ & $64.3(14.2)$ & $47.9(3.4)$ & $0.69(0.28)$ & $20.4(5.4)$ \\
\hline
\end{tabular}

\footnotetext{
* p-value one-way ANOVA $<0.001$

$\dagger$ p-value Chi-square: 0.126

$\ddagger$ p-value one-way ANOVA: 0.110

$\S$ p-value one-way ANOVA: 0.257

I| pooled data from 11 different regions with only one study pharmacy
} 
Table 3 Association between patient characteristics and time to the first test strips dispensed. In bold all determinants that are significantly associated $(\mathrm{p}<0.05)$ with the outcome in the adjusted model

\begin{tabular}{|c|c|c|c|}
\hline & unadjusted HR $(95 \% \mathrm{CI})$ & adjusted HR $(95 \% \mathrm{CI}) *$ & $\begin{array}{l}\text { pharmacy adjusted } \\
\operatorname{HR}(95 \% \mathrm{CI}) \dagger\end{array}$ \\
\hline $\begin{array}{l}\text { age at start of antidiabetic drug use } \\
\text { (per } 10 \text { years) } \\
\text { gender }\end{array}$ & $0.77(0.74-0.79)$ & $0.91(0.86-0.96)$ & $0.89(0.85-0.94)$ \\
\hline male $\{$ reference $\}$ & 1.00 & 1.00 & 1.00 \\
\hline female & $0.97(0.88-1.07)$ & $0.97(0.86-1.09)$ & $0.95(0.85-1.08)$ \\
\hline \multicolumn{4}{|l|}{ year of start of antidiabetic drug use } \\
\hline 1993 & $1.09(0.87-1.38)$ & $0.92(0.70-1.22)$ & $1.05(0.78-1.40)$ \\
\hline 1994 & $1.20(0.96-1.51)$ & $1.07(0.82-1.41)$ & $1.23(0.91-1.65)$ \\
\hline 1995 & $1.28(1.02-1.61)$ & $1.08(0.82-1.41)$ & $1.30(0.91-1.73)$ \\
\hline 1996 & $1.14(0.90-1.45)$ & $0.90(0.69-1.19)$ & $1.10(0.82-1.48)$ \\
\hline 1997 & $1.23(0.97-1.57)$ & $1.04(0.79-1.36)$ & $1.25(0.94-1.68)$ \\
\hline 1998 & $1.43(1.12-1.81)$ & $1.25(0.96-1.63)$ & $1.60(1.20-2.14)$ \\
\hline 1999 & $1.30(1.01-1.68)$ & $1.17(0.88-1.54)$ & $1.34(1.00-1.81)$ \\
\hline 2000 & $1.31(1.00-1.71)$ & $1.21(0.91-1.63)$ & $1.41(1.03-1.93)$ \\
\hline 2001 & $1.73(1.27-2.35)$ & $1.63(1.18-2.26)$ & $1.80(1.27-2.56)$ \\
\hline \multicolumn{4}{|l|}{ type of treatment } \\
\hline insulin use in type $1 \mathrm{DM}$ \{reference & 1.00 & 1.00 & 1.00 \\
\hline oral hypoglycemic agents (OHA) & $0.16(0.13-0.21)$ & $0.21(0.16-0.29)$ & $0.21(0.15-0.28)$ \\
\hline both insulin and OHA & $3.08(2.31-4.10)$ & $3.75(2.72-5.16)$ & $4.57(3.29-6.34)$ \\
\hline $\begin{array}{l}\text { only insulin in type } 2 \mathrm{DM} \\
\text { use of co-medication (ATC-code) }\end{array}$ & $1.49(1.11-1.98)$ & $1.83(1.34-2.50)$ & $2.30(1.65-3.21)$ \\
\hline diuretics (ATC-code C03, C07B or C07C) & $0.81(0.73-0.91)$ & $0.90(0.78-1.04)$ & $0.91(0.79-1.06)$ \\
\hline beta blockers (C07, except sotalol) & $0.87(0.78-0.97)$ & $1.03(0.90-1.18)$ & $0.98(0.85-1.13)$ \\
\hline ACE-inhibitors (C09) & $1.01(0.89-1.14)$ & $1.05(0.90-1.22)$ & $1.09(0.93-1.27)$ \\
\hline calcium antagonists (C08) & $0.94(0.82-1.08)$ & $1.04(0.87-1.23)$ & $0.97(0.93-1.27)$ \\
\hline cholesterol lowering drugs (C10A or B04A) & $0.98(0.83-1.15)$ & $0.96(0.79-1.15)$ & $0.97(0.80-1.17)$ \\
\hline corticosteroids ( $\mathrm{HO2}$ ) & $1.74(1.39-2.20)$ & $1.10(0.90-1.35)$ & $1.00(0.80-1.24)$ \\
\hline benzodiazepines (N05BA or N05CD) & $1.09(0.98-1.22)$ & $1.15(1.00-1.32)$ & $1.17(1.02-1.34)$ \\
\hline antidepressants (N06A) & $1.07(0.88-1.31)$ & $0.88(0.68-1.13)$ & $0.89(0.70-1.13)$ \\
\hline antipsychotics (N05A, except N05AN) & $1.15(0.87-1.52)$ & $0.94(0.66-1.33)$ & $0.98(0.70-1.38)$ \\
\hline
\end{tabular}

* adjusted for age, gender, year of start of antidiabetic drug use, type of treatment and all co-medication

$\dagger$ adjusted for age, gender, year of start of antidiabetic drug use, type of treatment, all co-medication and dispensing pharmacy

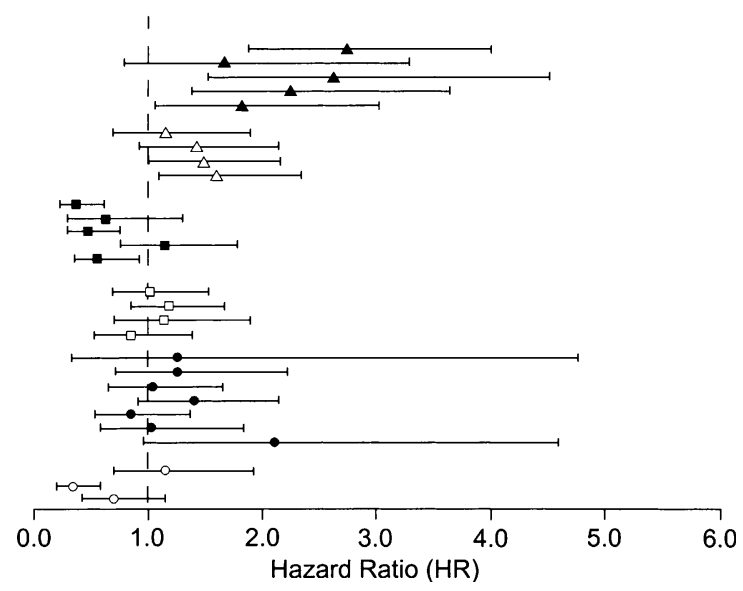

Fig. 1 Association between dispensing pharmacy and time to first test strips dispensed adjusted for age, gender, type of treatment, year of start of antidiabetic drug use and comedication, clustered by geographical region. Different symbols represent different regions. Data from regions with only one study pharmacy have been omitted (11 pharmacies) observations were in accordance with previous studies using frequency of SMBG as an outcome [11, 12, 17, 18], although comparative data on the effect of age and gender were inconclusive.

Arday et al. found large variations in diabetes care between US states, that remained after adjustment for characteristics of state residents [13]. Although we performed our study in much smaller geographical areas, we observed a similar regional effect. Regional variation in rates of health care interventions is not restricted to diabetes care. Geographical variation has also been reported in, for example, treatment of acute myocardial infarction and surgical procedures such as knee arthoplasty [19, 20]. Although many different explanations for these geographic variations are proposed, it is believed that much variability is associated with differences in individual clinical practice [21]. SMBG among insulin users nears $100 \%$, making physicians, preferences a relative unimportant factor. However, practice differences in the distribution of 
testing materials could be relevant. In our study, region correlates well with different hospital referral areas. The key decision maker in choice of self-monitoring equipment - diabetes nurse specialist - is often located in the hospital setting. Their practice pattern, for example based on collaboration with a certain supplier of testing materials, would affect dispensing of test strips of all community pharmacies within the referral area.

In some regions, we still observed significant differences between dispensing pharmacy in the use of test strips after adjustment for variation in patient population. This suggests that pharmacy characteristics may have an effect on the proportion of test strip users. Although we had no additional information of these characteristics, previous studies report for example physical lay-out of the pharmacy, computer support, knowledge and competence as well as lack of time and reimbursement as barriers to implementation of pharmaceutical care activities [22, 23].

In the Netherlands, patients can acquire test strips through other distribution channels than community pharmacies, such as mail order or from diabetes nurses in specialised clinics. Since type 1 patients - in contrast to type 2 patients - are almost always treated by a specialist, the structure of diabetes care may differ. Therefore, it is possible that type 1 patients are less likely to receive test strips in a community pharmacy. This would result in a relatively higher probability of test strips dispensed to type 2 patients. The third party distribution also explains the finding that only one out of five patients receive test strips through community pharmacy. Moreover, the low proportion of test strip use may also be explained by the inclusion of incident patients only and a median follow-up of 2.1 years.

The lack of information on distribution of test strips through other channels does not affect the generalisability of our study. Since we are interested in differences between pharmacies, it is not necessary to have data on every patient using test strips. Moreover, from reports of the Foundation of Pharmaceutical Statistics (SFK) we estimate that about $60 \%$ of the costs of SMBG are made in the community pharmacy, suggesting that our data still represent a large proportion of test strip users [24].

A limitation of our study is the relative low proportion of type 1 patients. Of all prevalent cases of diabetes in the Netherlands, about $10-15 \%$ have type 1 diabetes [25]. This discrepancy is due to the definition of an incident patient: at least 1 year of dispensing history before the first antidiabetic drug prescription is filled. Since type 1 patients have in general less comorbidity at the date of diagnosis and are younger at time of diagnosis, they are less likely to be included as incident patient than type 2 patients. This lowers the proportion of type 1 patients in the study population.
However, if we had included prevalent users of antidiabetic drugs, misclassification of insulin users would have occurred, because about $25 \%$ of all type 2 patients use only insulin therapy to control their hyperglycemia. Moreover, the association between type of antidiabetic treatment and use of test strips is not likely to be influenced by this exclusion of some type $1 \mathrm{pa}-$ tients. For the observed differences between pharmacies, this would be even less important.

Since diabetic patients visit a pharmacy on an average of five to six times per year, community pharmacists are well placed to educate patients on diabetes. Dispensing of test strips provides an opportunity to support the patient with the complicated process of SMBG. Furthermore, pharmacist-led intervention programs, including education and training of SMBG skills, showed a decrease in HbAlc-values attributed to the pharmaceutical care services [26-30]. Moreover, because of the observed differences between pharmacies, not all may experience the same barriers in dispensing of test strips. Since about $60 \%$ of all Dutch community pharmacies are owned by an individual pharmacist [31], this implies a significant effect of region-specific factors in the structure and process of the activities of the individual pharmacists.

For practice research, our findings suggest that the external validity of studies into dispensing of test strips might be limited if geographical variation is not taken into consideration. This might be true for other community pharmacy activities, especially those in which other health care providers also play a role. Therefore, it is important for studies on pharmaceutical care activities to include a representative sample of community pharmacies.

Although our study implies that regional characteristics are important in dispensing of test strips, our dataset did not provide information to further investigate these factors. Future research should focus on elucidation of key factors in the dispensing process of testing material. This would facilitate the evidencebased implementation of diabetes care activities in community pharmacy.

\section{Conclusion}

In conclusion, differences between pharmacies in the rate of dispensing of test strips are independent of differences in patient characteristics among pharmacies. However, this association depends on the geographical region of the pharmacy. Therefore, implementing practice guidelines for diabetes care in community pharmacies, will require different approaches in different regions. This study also underscores the complexity of determining relevant quality indicators for providing support to patients practising SMBG. 
Acknowledgements This study was supported by an unrestricted grant from the Royal Society for the Advancement of Pharmacy (KNMP), the Hague, the Netherlands.

\section{References}

1. Stratton IM et al. Association of glycemia with macrovascular and microvascular complications of type 2 diabetes (UKPDS 35): prospective observational study. BMJ 2000; 321:405-412

2. The DCCT Research Group. The effect of intensive treatment of diabetes on the development and progression of long-term complications insulin-dependent diabetes mellitus. NEJM 1993; 329:977-986

3. American Diabetes Association. Standards of medical care in diabetes. Diab Care 2004; 27:S15-S35

4. Wiersma TJ, Heine RJ, Rutten GHE. NHG-Standard Diabetes mellitus type 2: Tweede herziening. Huisarts Wet 2006; 49:137-152

5. American Diaberes Association. Tests of glycemia in diabetes. Diab Care 2004; 27:S91-S93

6. EuroPharmForum (2001) Improved quality in diabetes care: the pharmacist in St Vincent team: protocol and guidelines. http://www.euro.who.int/document/e75680.pdf (6 Jun. 2005)

7. Practice Guidance for Community Pharmacists on the Care of People with Diabetes. London: Royal Pharmaceutical Society of Great Britain. Nov 2004. http://www.rpsgb.org/ pdfs/diabguid3.pdf\#xml = http://www.rpsgb.org/search/pdfhi.php? all $=1 \&$ filepath $=. . /$ pdfs/diabguid3.pdf\&search $=$ diabetes (6 Jun. 2005)

8. American Society of Hospital Pharmacists (ASHP) (1995) Therapeutic position statement on strict glycemic control in selected patients with insulin-dependent diabetes mellitus. Am J Health-Syst Pharm 52: 2709-2711

9. WINAp (2000) FPZ Standaard Diabetes Mellitus Type 2 [Pharmaceutical Care Standard Diabetes Mellitus Type 2]. Den Haag: WINAp

10. Storimans MJ, Talsma $\mathrm{H}$, Klungel $\mathrm{OH}$, Blaey CJde. Dispensing glucose test materials in Dutch community pharmacies. Pharm World Sci 2004; 32:52-55

11. Scorpiglione $N$ et al. Epidemiology and determinants of blood glucose self-monitoring in clinical practice. Diabetes Res Clin Pract 1996; 34:115-125

12. Karter AJ, Ferrara A, Darbinian JA, Ackerson LM, Selby JV. Self-monitoring of blood glucose: language and financial barriers in a managed care population with diabetes. Diab care 2000; 23:477-483

13. Arday DR, Fleming BB, Keller DK. Variation in diabetes care among states: do patient characteristics matter? Diab Care 2002; 25:2230-2237

14. Herings R, Stricker B, Boer Ade, Bakker A, Sturmans F. Benzodiazepines and the risk of falling leading to femur fractures. Dosage more important than elimination halflife. Arch Intern Med 1995; 155:1801-1807
15. Herings R, Boer Ade, Stricker B, Leufkens H, Porsius A. Hypoglycaemia associated with use of inhibitors of angiotensin converting enzyme. Lancet 1995; 345:1195-1198

16. Erkens JA et al. Cardiovascular drug use and hospitalisations attributable to type 2 diabetes mellitus. Diab Care $2001 ; 24: 1428-1432$

17. Zgibor JC, Simmons D. Barriers to blood glucose monitoring in a multiethnic community. Diab Care 2002; 25:1772-1777

18. Harris MI, Cowie CC, Howie LJ. Self-monitoring of blood glucose by adults with diabetes in the United States population. Diab care 1993; 16:1116-1123

19. O'Connor GT et al. Geographic variation in the treatment of acute myocardial infarction: the Cooperative Cardiovascular Project. JAMA 1999; 281:627-633

20. Skinner J, Weinstein JN, Sporer SM, Wennberg JE. Racial, ethnic, and geographic disparities in rates of knee arthroplasty among medicare patients. New Eng J Med 2003; 349:1350-1359

21. Wennberg JE. Understanding geographic variations in health care delivery. New Eng J Med 1999; 340:52-53

22. Bell HM, McElnay JC, Hughes CM. A qualitative investigation of the attitudes and opinions of community pharmacists to pharmaceutical care. J Soc Adm Pharm 1998; $15: 284-295$

23. Odedina FT, Segal R, Hepler CD. Providing pharmaceutical care in community practice: differences between providers and non-providers of pharmaceutical care. J Soc Adm Pharm 1995; 12:170-180

24. Stichting Farmaceutische Kengetallen (2001) Farmacie in cijfers: toename in gebruik hulpmiddelen [Pharmacy in figures: rise in use of medical aids]. Pharm Weekbl 136: 829

25. Lutterman J (2002) Farmacotherapie bij diabetes mellitus: huidige situatie en toekomstverwachtingen [Pharmocotherapy of diabetes mellitus: current situation and projections]. In: Timmerman $\mathrm{H}$, van den Bery Jeths A, editors. Volksgezondheid toekomst verkenningen. Bilthoven: RIVM. ISBN 90-313-3702-1

26. Cranor CW, Bunting BA, Christensen DB. The Asheville Project: long-term clinical and economic outcomes of a community pharmacy diabetes care program. J Am Pharm Assoc (Wash) 2003; 43:173-184

27. Cranor CW, Christensen DB. The Asheville Project: shortterm outcomes of a community pharmacy diabetes care program. J Am Pharm Assoc (Wash) 2003; 43:149-159

28. Berringer $\mathrm{R}$ et al. Outcomes of a community pharmacybased diabetes monitoring program. J Am Pharm Assoc (Wash) 1999; 39: 791-797

29. Cioffi ST, Caron MF, Kalus JS, Hill P, Buckley TE. Glycosylated hemoglobin, cardiovascular, and renal outcomes in a pharmacist-managed clinic. 2004; 38: 771-775

30. Wermeille J, Bennie M, Brown I, McKnight J. Pharmaceutical care model for patients with type 2 diabetes: integration of the community pharmacist into the diabetes team - a pilot study. Pharm World Sci 2004; 26: 18-25

31. Stichting Farmaceutische Kengetallen (2003) Weer meer apotheken [Again, more pharmacies]. Pharm Weekbl 138: 128 